\title{
Single Step Hybrid Coating Process to Enhance the Electrosteric Stabilization of Inorganic Particles
}

\author{
Chantal Rufier, ${ }^{\dagger}$ Mathias Reufer, ${ }^{\dagger}$ Hervé Dietsch, ${ }^{*,+}$ and Peter Schurtenberger, ${ }^{*,+}$ \\ ${ }^{\dagger}$ Adolphe Merkle Institute and Fribourg Center for Nanomaterials, University of Fribourg, Route de l'Ancienne Papeterie P.O. Box 209, \\ 1723 Marly 1, Switzerland \\ ${ }^{\ddagger}$ Physical Chemistry, University of Lund, S-22100 Lund, Sweden
}

\begin{abstract}
We report on a single-step coating process and the resulting colloidal stability of silica-coated spindle-type hematite nanoparticles (NPs) decorated with a layer of poly(acrylic acid) (PAA) polyelectrolyte chains that are partially incorporated into the silica shell. The stability of PAA coated NPs as a function of $\mathrm{pH}$ and salt concentration in water was compared to bare hematite particles and simple silica-coated hematite NPs, studying their electrophoretic mobility and the hydrodynamic radius by dynamic light scattering. Particles coated with this method were found to be more stable upon

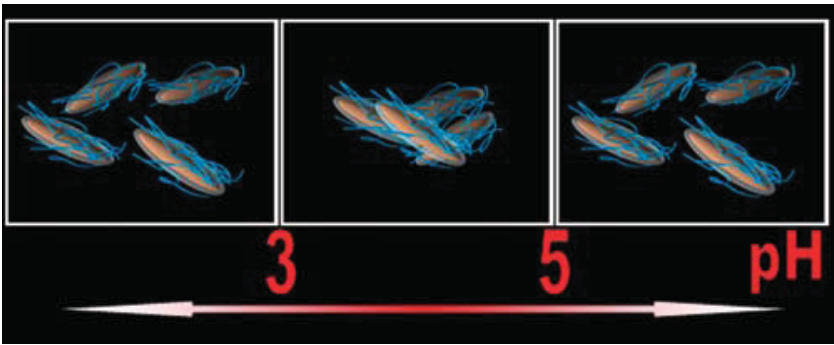
the addition of salt at $\mathrm{pH} 7$, and their aggregation at the $\mathrm{pH}$ of the isoelectric point is reversible. The hybrid coating appears to increase the colloidal stability in aqueous media due to the combination of the decrease of the isoelectric point and the electrosteric stabilization. This coating method is not limited to hematite particles but can easily be adapted to any silica-coatable particle.
\end{abstract}

\section{INTRODUCTION}

Iron oxide nanoparticles have attracted considerable interest due to the tunable nature of their magnetic properties and shape, ${ }^{1-6}$ which makes them attractive for a host of potential medical applications, $^{7-9}$ such as protein and cell separation, ${ }^{6,10}$ drug delivery, ${ }^{11}$ and use as magnetic contrast agent. ${ }^{12}$ In our study, we focus on spindle-type NPs made of hematite $\left(\alpha-\mathrm{Fe}_{2} \mathrm{O}_{3}\right)$, the most stable phase of iron oxide in air at ambient conditions. It is only weakly ferromagnetic, so that dipole-dipole interactions do not induce aggregation. It has been shown that the spindle-type particles align perpendicular to an external magnetic field, ${ }^{13}$ which might have considerable consequences for current attempts to create fieldcontrolled nanostructures and novel nanocomposite materials (i.e., aligned nanoparticles in polymer matrix) with anisotropic properties. An essential parameter for applications is the colloidal stability of NPs in water, which is mainly determined by the $\mathrm{pH}$ and ionic strength of a suspension. ${ }^{14-17}$ Colloidal stability depends on the balance of the repulsive and attractive forces that exist between particles in suspension. Controlling the surface chemistry of the colloids, using either a steric and electrostatic stabilization approach, can avert aggregation of NPs. In the steric stabilization mechanism, a coated layer hinders the particles from coming too close by, and creates a repulsive interaction. ${ }^{17,18}$ The van der Waals attraction that otherwise acts at close proximities is thus limited, which prevents the particles from aggregation. In the electrostatic mechanism, the particles are charged, thus inducing long-range Coulomb repulsion. A disadvantage of an electrostatic repulsion is that the $\mathrm{pH}$ and ionic strength of a suspension strongly influence the colloidal stability, where salt screens the surface charges and consequently lowers the electrostatic barrier. The charge density on particles depends on $\mathrm{pH}$, and at the isoelectric point, the positive and negative charges compensate each other leading to an overall neutral particle. At the isoelectric point, there is no more electrostatic stabilization and particles can aggregate. The isoelectric point of hematite particles is at $\mathrm{pH}=7$, which is problematic for experiments in aqueous media at neutral $\mathrm{pH}$ and applications in biological with physiological conditions: $150 \mathrm{mM}$ of $\mathrm{NaCl}$ and $\mathrm{pH}=7.4$. Therefore, surface modifications are necessary to ensure stability under these conditions.

When considering such modifications, there exist a number of organic as well as inorganic coatings. Among the organic compounds, anionic surfactants, such as sodium 4-octylbenzenesulfonate, ${ }^{19}$ sodium oleate, or phosphates, ${ }^{5,6}$ are usually used to stabilize iron oxide particle dispersions. Alternatively, the surface of iron nanoparticles can be decorated with acidic ligands in order to obtain stable ferrofluids over a large $\mathrm{pH}$ range. ${ }^{20}$ Polymer coatings also provide stability for iron oxide particles, where the long polymer chains induce steric stabilization. Some commonly used nonionic polymers are poly(vinyl alcohol) (PVA), ${ }^{21}$ poly(vinyl pyrrolidone) (PVP), poly(ethylene glycol) (PEG), and dextran. ${ }^{22}$ In the case of polyelectrolytes, such as poly (acrylic acid $)^{23}$ and chitosan, the steric stabilization is coupled with an electrostatic stabilization which in turn promotes particle dispersion in aqueous environment. For example, layer by layer 


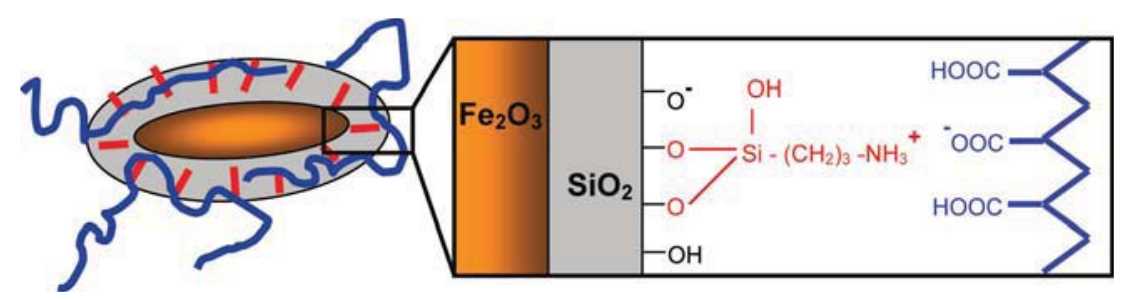

Figure 1. Schematic representation of the PAA/silica-coated hematite particle.

( $\mathrm{LbL}$ ) stabilization of colloids can be achieved by stepwise adsorption of oppositely charged polyelectrolyte (PE) ${ }^{24}$ Alternatively, amphiphilic block copolymers, such as poly(styrene- $b$-styrene sulfonate), can be used as a stabilizer for emulsion or dispersion polymerization of latexes. ${ }^{25}$ However, a persisting problem of organic stabilization is that the compounds are physically adsorbed on the particle surface and can be removed by dilution or washing.

Inorganic shells can also be applied to existing particles. For instance, Plaza et al. and Matijevic et al. modified iron oxide particles with an yttrium or manganese oxide coating, respectively. ${ }^{16,26}$ However, these coatings shifted the isoelectric point from $\mathrm{pH} 7$ to $\mathrm{pH} 8$ or 5 , making the core-shell particles aggregate at these $\mathrm{pH}$ values which are again too close to neutral $\mathrm{pH}$. Several authors have reported on iron oxide nanoparticles with silica coatings that take advantage of the charged surface in water at $\mathrm{pH}$ higher than $4 .{ }^{4,27-29}$ The surface properties of core-shell particles correspond to the shell material properties; thus, surface modification using silane coupling agents is analogous to the chemistry used for silica particle modification. ${ }^{30}$ Therefore, it is possible to combine both organic and inorganic coatings to take advantage of the electrostatic repulsion from the silica shell and the steric repulsion due to an organic compound. Tsang et al. immobilized $\beta$-lactamase onto the silicaencapsulated nanoparticles via a surface modification with 3 -aminopropyltriethoxysilane. ${ }^{31}$ Butterworth et al. polymerized pyrrole from silica-coated magnetite. ${ }^{32}$ Dagallier et al. combined silica-coated hematite nanoparticles with a thermosensitive polymer shell. ${ }^{33}$ However, these modifications require three synthesis steps from the iron particle to the surface-modified silicated hematite particles.

Previously, Graf and co-workers ${ }^{34}$ developed a silica coating method for nanoparticles, Sacanna et al. ${ }^{35}$ incorporated fluorescent dye in the silica shell by a co-condensation of two silicate reagents, and Wang et al. ${ }^{36}$ studied the infiltration of polyelectrolyte into nanoporous silica particles. The present work combines the three previous studies to develop a suitable method to strongly increase the stability of ellipsoidal hematite iron oxide nanoparticles dispersed in water. We report on the novel process of coating hematite particles with silica and a polyelectrolyte in a single step. Hematite nanoparticles were stabilized by co-condensation of a silicate reagent and a positively charged silane agent in the presence of a negatively charged polyelectrolyte. Figure 1 is a representation of the proposed surface treatment onto spindle-type hematite particles, which were selected as an interesting anisotropic orientable colloidal particle system. The surface modifications were characterized by TEM, infra-red spectroscopy, zeta potential measurement, dynamic light scattering (DLS), and SAXS.

\section{EXPERIMENTAL SECTION}

2.1. Materials. Iron(III) perchlorate hexahydrate $\mathrm{Fe}\left(\mathrm{ClO}_{4}\right)_{3} \cdot 6 \mathrm{H}_{2} \mathrm{O}$, polyvinylpyrrolidone (PVP, $10000 \mathrm{~g} / \mathrm{mol}$ ), tetramethylammonium hydroxide (TMAH) solution 25\% in water, and poly(acrylic acid) (PAA,
$100000 \mathrm{~g} / \mathrm{mol}$ ) $35 \mathrm{wt} \%$ in water were all provided by Sigma Aldrich and used as received. Sodium dihydrogen phosphate monohydrate $\mathrm{NaH}_{2}$ $\mathrm{PO}_{4} \cdot \mathrm{H}_{2} \mathrm{O}$ and urea from Fluka, tetraethyl orthosilicate (TEOS) from Merck, 3-aminopropyl tetraethoxysilane (APTES) from ABCR, and absolute ethanol from Fluka were also used without further purification.

Ultrapure water (18.2 M $\Omega . c m)$ purified by a Milli-Q system was used throughout the experiments.

2.2. Hematite Nanoparticle Synthesis. Anisotropic hematite nanoparticles were synthesized based on the method described by Ocaña et al. ${ }^{2}$ In a typical synthesis, a $2 \mathrm{~L}$ glass bottle was charged with $92.4 \mathrm{~g}$ of $\mathrm{Fe}\left(\mathrm{ClO}_{4}\right)_{3} \cdot 6 \mathrm{H}_{2} \mathrm{O}(200 \mathrm{mmol}), 1.3 \mathrm{~g}$ of $\mathrm{NaH}_{2} \mathrm{PO}_{4} \cdot \mathrm{H}_{2} \mathrm{O}(9.4$ mmol, i.e., molar ratio $\left.\mathrm{NaH}_{2} \mathrm{PO}_{4} / \mathrm{Fe}^{3+}=0.047\right)$, and $12 \mathrm{~g}$ of urea $\left(\mathrm{NH}_{2}\right)_{2} \mathrm{CO}(0.2 \mathrm{~mol})$ in $2 \mathrm{~L}$ of ultra pure water, and then sonicated until a homogeneous solution resulted. The solution was kept in a preheated oven at $98{ }^{\circ} \mathrm{C}$ for $24 \mathrm{~h}$. Approximately $2 \mathrm{~g}$ of a brown precipitate was obtained in a brown solution. The supernatant was decanted and the precipitate was cleaned by seven successive cycles of washing with pure water and centrifugation at $13000 \mathrm{rpm}$ for $10 \mathrm{~min}$ to remove the impurities. Finally, ca. $2 \mathrm{~g}$ of hematite nanoparticles was recovered and dispersed into $40 \mathrm{~mL}$ of water. Particle size and distribution were measured from TEM pictures of about 200 particles. The average particle length $a$ and width $b$ are $262 \pm 33 \mathrm{~nm}$ and $57 \pm 5 \mathrm{~nm}$, respectively with an axial ratio $a / b=4.5 \pm 0.5$. (see Figure $1 S$ in Supporting Information for the size distribution).

2.3. PAA and Silica Coating of Hematite Nanoparticles. The simple silica coating and the combined PAA-silica coating were performed using the same conditions with the exception of the addition of APTES and PAA in the latter procedure. The particle concentration and the quantity of TEOS were identical. To stabilize the particles during the coating, PVP was adsorbed onto the surface of hematite NPs, using the following procedure: In a $1 \mathrm{~L}$ container, $100 \mathrm{mg}$ of spindle hematite NPs and $2 \mathrm{~g}$ of PVP were dispersed in $220 \mathrm{~mL}$ of pure water. The particles were sonicated for $2 \mathrm{~h}$ and then stirred for $15 \mathrm{~h}$. The nanoparticles were then washed by water dispersion followed by $13000 \mathrm{rpm}$ centrifugation for $10 \mathrm{~min}$. The PVP stabilized hematite NPs were dispersed in $10 \mathrm{~mL}$ of water. The NP suspension (i.e., containing $100 \mathrm{mg}$ of hematite NPs) was then added to ethanol $(225 \mathrm{~g})$ in a $500 \mathrm{~mL}$ plastic bottle followed by sonication and mechanical stirring ( $400 \mathrm{rpm}) .85 \mathrm{mg}$ of TMAH in $3 \mathrm{~mL}$ of ethanol was added to the nanoparticle suspension. After $30 \mathrm{~min}$ of stirring, $250 \mu \mathrm{L}$ of TEOS in $1 \mathrm{~mL}$ of ethanol was added at once. A mixture of $20 \mu \mathrm{L}$ of APTES and $750 \mu \mathrm{L}$ of TEOS in $3 \mathrm{~mL}$ of ethanol was then injected in 3 portions every $30 \mathrm{~min}$. The suspension was allowed to stand for $30 \mathrm{~min}$, and then PAA $(100 \mathrm{~kg} / \mathrm{mol})(270 \mu \mathrm{L}$ in $1 \mathrm{~mL}$ of ethanol $)$ was added. Sonication was applied for two hours after the last injection, and the mechanical stirring was further continued overnight at room temperature.

2.4. Characterization. Infrared spectroscopy was done on dried samples $\left(3 \mathrm{~h}\right.$ at $100{ }^{\circ} \mathrm{C}$ ) with a FT-IR Spectrometer Tensor 27 from Bruker with a golden gate.

A transmission electron microscope (TEM-CM100, Philips) operating at $80 \mathrm{keV}$ was used for size and morphology studies of the synthesized particles. A typical sample for TEM was prepared by concentrating the particle dispersion using a lab centrifuge at $10000 \mathrm{rpm}$ and drying one droplet of $1 \mathrm{vol} \%$ of particles onto a carbon-coated ( $300 \mathrm{mesh}$ ) grid. 


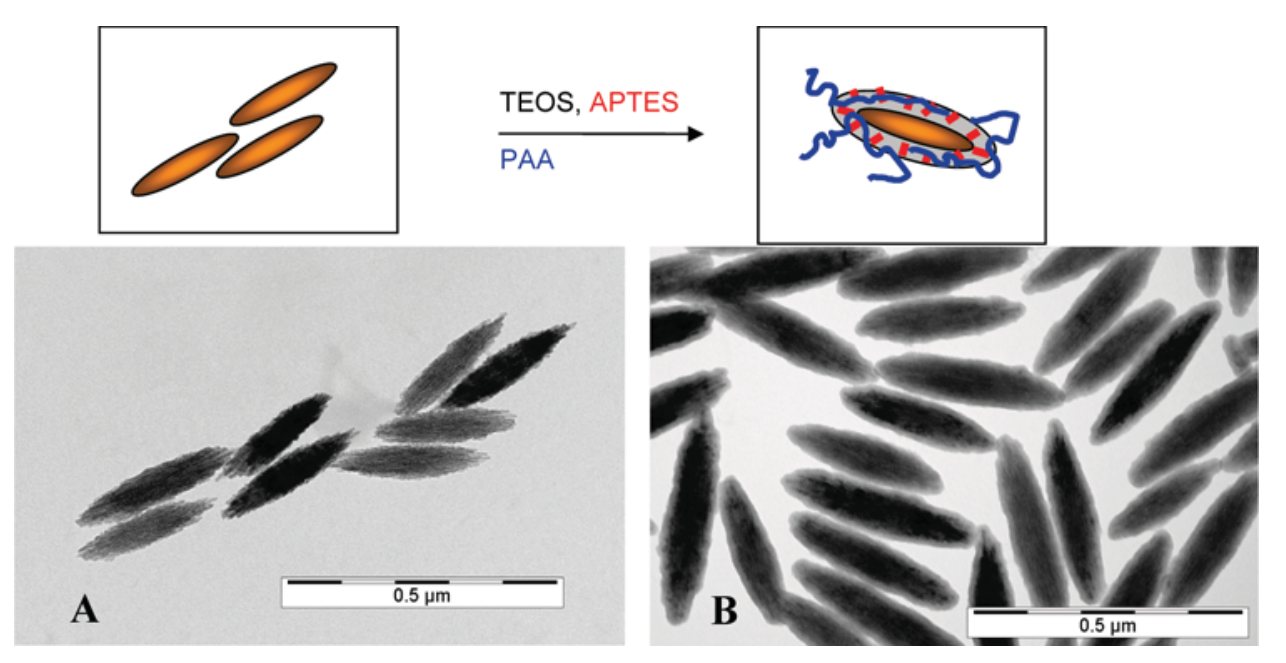

Figure 2. TEM pictures of ellipsoidal hematite NPs before (A) and after (B) the coating.

The electrophoretic mobility of the particles was determined at $25{ }^{\circ} \mathrm{C}$ using a Zetasizer Nano-Z from Malvern Instruments. The results shown represent an average from at least three individual measurements, and error bars given correspond to the standard deviation. The $\mathrm{pH}$ dependence was investigated in dilute suspensions $(0.01 \mathrm{~g} / \mathrm{L})$ and the $\mathrm{pH}$ was adjusted adding $\mathrm{NaOH}$ or $\mathrm{HCl}(0.1 \mathrm{M})$. The same suspensions were used for dynamic light scattering (DLS) measurements performed on a Goniometer System from LS Instrument, Switzerland, equipped with a HeNe-Laser operating at a wavelength of $632.8 \mathrm{~nm}$. The average particle hydrodynamic radii were determined at a scattering angle of $90^{\circ}$ and a temperature of $25^{\circ} \mathrm{C}$. We also performed DLS for scattering angles in the range $10-90^{\circ}$ and found a linear increase of the decay rate with the square of the scattering vector. This rules out contributions of rotational diffusion due to the anisotropic particle shape that might affect data at high scattering angles.

Small angle X-ray scattering (SAXS) were carried out at the cSAXS beamline at the Swiss Light Source (Paul Scherrer Institute). We used the $11.1924 \mathrm{keV}$ harmonic corresponding to a wavelength of $\lambda=$ $0.11078 \mathrm{~nm}$. All measurements were performed in quartz capillaries of $1 \mathrm{~mm}$ diameter $\left(0.1 \mathrm{~mm}\right.$ wall thickness) at $24^{\circ} \mathrm{C}$. The particles were dispersed in water at a concentration of approximately $0.5 \mathrm{vol} \%$.

\section{RESULTS AND DISCUSSION}

In this work, ellipsoidal hematite nanoparticles were synthesized and then coated with silica and poly(acrylic acid) to stabilize the hematite NPs in aqueous dispersion. To attract the negatively charged polyelectrolyte on the silica shell, the TEOS was co-condensated with APTES on hematite NPs in ethanol. The amino groups destabilized the particles changing the charge density, so the PAA was introduced $30 \mathrm{~min}$ after the last addition of silicate reagents to prevent aggregation. The PAA chains interact with the APTES amino groups and allow us to develop a single-step coating process, limiting the multiple cleaning of the excess of reagents. Using this single-step process, the polyelectrolyte is contained in the silica shell leading to a hybrid shell and an electrosteric stabilization of the particles.

The NPs were characterized by TEM and infrared spectroscopy (IR). TEM images of particles before and after the cocondensation coating (Figure 2) show a silica layer of about $10 \mathrm{~nm}$. However, the PAA was not visible in TEM. IR spectroscopy was used to observe the presence of the polyelectrolyte. Figure 3 shows the IR spectra from 1000 to $2000 \mathrm{~cm}^{-1}$ for PAA and the particles before coating, with a simple silica coating and with a silica coating in the presence of PAA (see Figure $2 S$ in Supporting

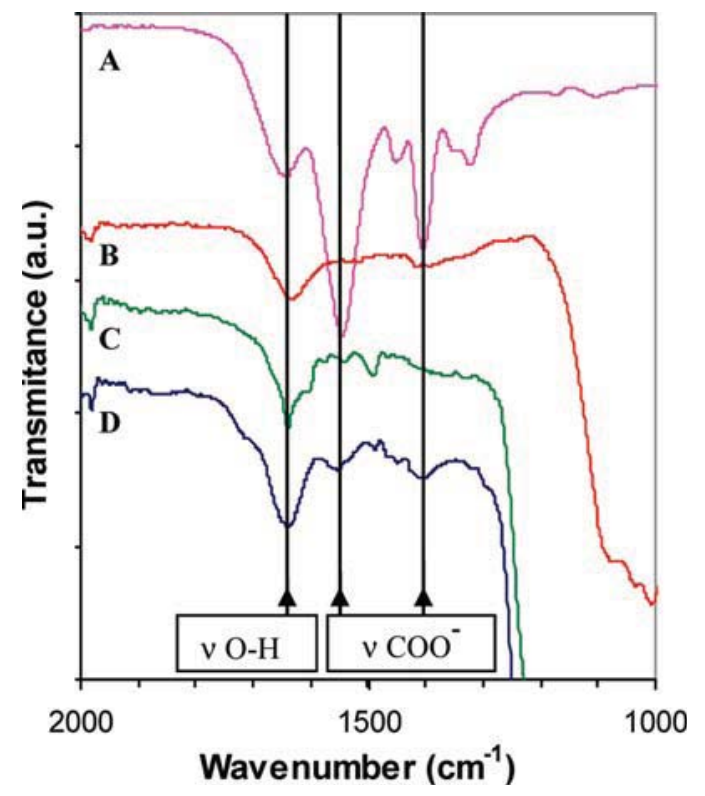

Figure 3. Overlay of infrared spectra of PAA in water (A, pink), hematite nanoparticles (B, red), silicated hematite NPs (C, green), and PAA-silicated hematite NPs (D, blue).

Information for the full spectra). In a $\mathrm{pH} 8$ solution, the carboxyl groups on PAA are deprotonated, and the bands at 1550 and $1400 \mathrm{~cm}^{-1}$ are characteristic of the carboxylate stretch. The PAAsilicated hematite NP spectrum shows the carboxylate characteristic bands from PAA proving the presence of the polymer.

The stability of the PAA-silica coating was checked after prolonged storage and repeated washing cycles. The sample was analyzed by IR spectroscopy directly after coating and stored for three months. After a new washing step with three centrifugation and water dispersion cycles, the particles were reanalyzed. The IR spectroscopy results are compared in Figure $3 \mathrm{~S}$ in the Supporting Information. Both IR spectra show the presence of the characteristic band of the carboxylate stretch from the PAA. This clearly shows the stability of the PAA coating even after three months of storage and repeated cycles of centrifugation and washing. 
The silicated particles with and without PAA were also characterized with SAXS. The experimental scattering intensities are shown in Figure 4. A quantitative analysis of the SAXS scattering curves with an appropriate model allows for the determination of the particle dimensions. Here, we used a model of polydisperse ellipsoids with a core-shell structure. Polydispersity was incorporated by using a discrete log-normal distribution for the core width and shell thickness with nine and five different grid values, respectively. We assumed a hematite-core scattering length density of $41.6 \times 10^{-6} \AA^{-2}$ and a density of $5.3 \mathrm{~g} / \mathrm{cm}^{3}$. The shells of 19.2 and $11.1 \mathrm{~nm}$ for the simple silica-coated particles and the PAAsilicated hematite NPs, respectively, gave values of $12.6 \times 10^{-6} \AA^{-2}$ and $13.0 \times 10^{-6} \AA^{-2}$ for the scattering length density (SLD), which correspond to silica densities of 1.47 and $1.52 \mathrm{~g} / \mathrm{cm}^{3}$. These densities are slightly lower than the value of $2 \mathrm{~g} / \mathrm{cm}^{3}$ expected for Stöber like silica, ${ }^{37}$ and indicate that the silica shell is porous. These results are comparable to results previously published by Reufer et al. on silica-coated hematite particles. ${ }^{38}$ The parameters used for fitting are summarized in Table 1 . They are very close to the characteristics obtained from the TEM picture analysis, indicating that no aggregation occurred in the suspensions.

The silica coating was added to the NPs to shift the isoelectric point and thus enhance the stability of the particles at neutral $\mathrm{pH}$. To determine the particle stability, the electrophoretic mobility

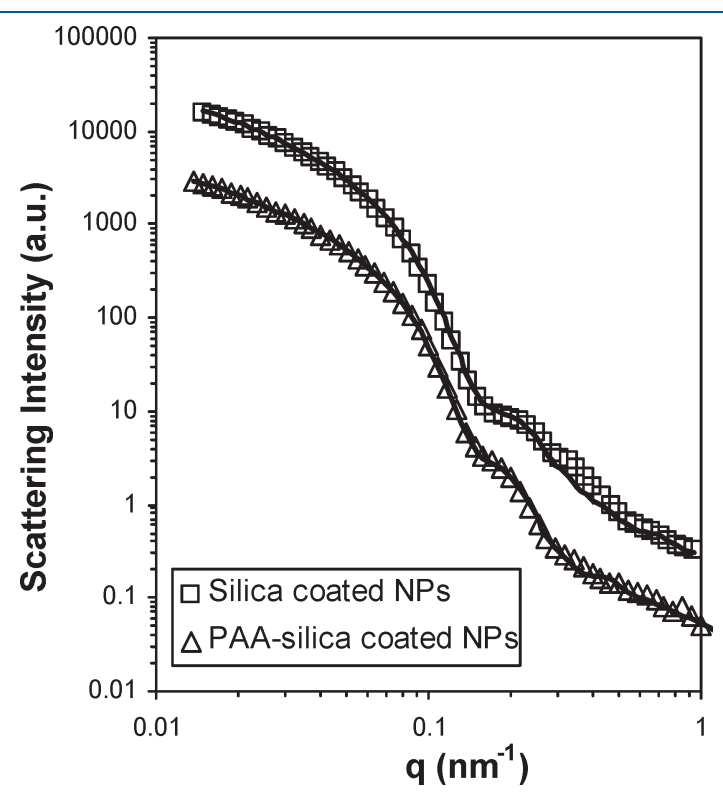

Figure 4. Scattering intensities of silicated hematite particles (squares) and PAA silicated hematite particles (triangles) at $0.5 \%(\mathrm{v} / \mathrm{v})$ in water, together with the corresponding fitted curves using a form factor for ellipsoidal core-shell particles (black lines; see text for details). The data curves are vertically shifted for clarity. and the hydrodynamic radius were determined as a function of $\mathrm{pH}$ at a particle concentration of $0.01 \mathrm{~g} / \mathrm{L}$ and at $25{ }^{\circ} \mathrm{C}$ (Figure 5). On the basis of the electrophoretic mobility measurements, the isoelectric point of the bare hematite nanoparticles is between 7 and 8 . At $\mathrm{pH}$ values sufficiently different from the isoelectric point, the particles are stabilized by electrostatic repulsions. The associated hydrodynamic radii obtained by DLS were ca. $100 \mathrm{~nm}$. When the suspension is unstable, aggregates form and the hydrodynamic radius increases dramatically. For $\mathrm{pH}>6$, aggregates of hematite particles were

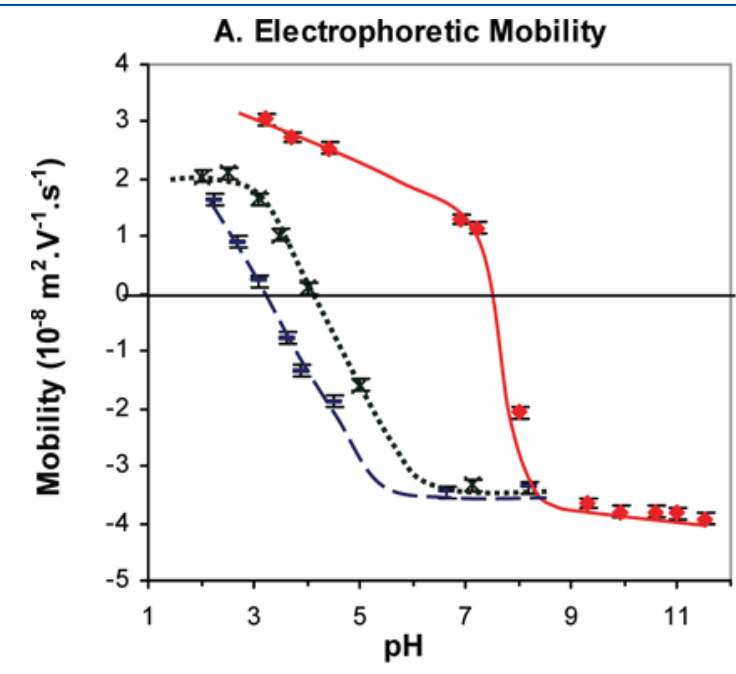

B. Hydrodynamic radius $\mathrm{Rh}$ (from DLS)

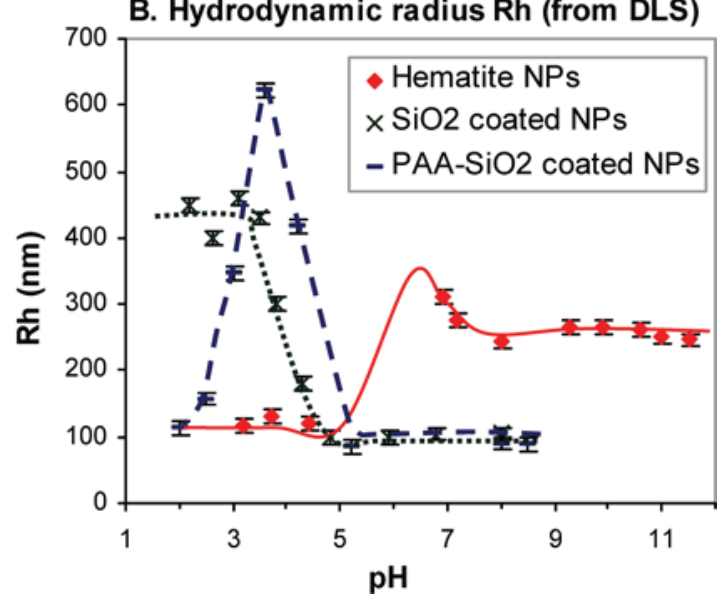

Figure 5. Electrophoretic mobility (A) and hydrodynamic radius $R_{\mathrm{h}}$ (B) vs $\mathrm{pH}$ for ellipsoidal hematite NPs ( $\bullet$, solid line), silicated hematites NPs ( $\times$, dotted line), and PAA-silicated hematite NPs (-, dashed line). Lines are guides for eye only. Particle concentration is $0.01 \mathrm{~g} / \mathrm{L}$. The $\mathrm{pH}$ was adjusted by the addition of aqueous solutions of $\mathrm{NaOH}$ and $\mathrm{HCl}$ $(0.1 \mathrm{~N})$.

Table 1. Particle Size Determined by TEM and SAXS

\begin{tabular}{|c|c|c|c|c|c|c|}
\hline \multirow[b]{2}{*}{ particles } & \multicolumn{3}{|c|}{ TEM } & \multicolumn{3}{|c|}{ SAXS } \\
\hline & length (nm) & width (nm) & silica thickness (nm) & length (nm) & width $(\mathrm{nm})$ & silica thickness $(\mathrm{nm})$ \\
\hline Hematite NPs & $262 \pm 33$ & $57 \pm 5$ & 0 & - & - & - \\
\hline Silicated hematites NPs & $330 \pm 55$ & $88 \pm 11$ & $15 \pm 2$ & $350^{a}$ & $91 \pm 7$ & $19 \pm 1$ \\
\hline PAA-silicated hematite NPs & $339 \pm 38$ & $75 \pm 6$ & $10 \pm 1$ & $350^{a}$ & $76 \pm 7$ & $11 \pm 3$ \\
\hline
\end{tabular}

${ }^{a}$ Length fixed for the fitting model. 


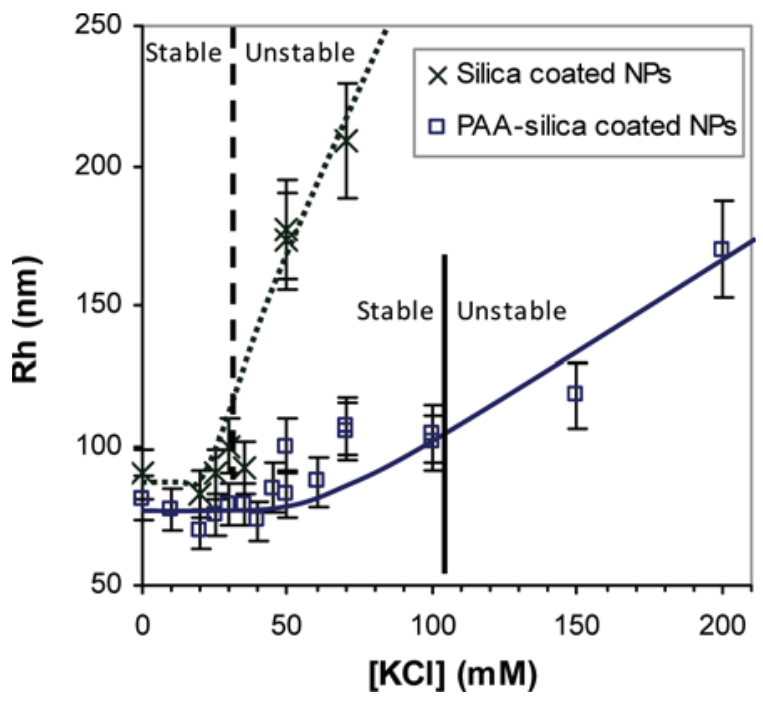

Figure 6. Influence of the salt concentration on the hydrodynamic radius $R_{\mathrm{h}}$ for silicated hematites NPs $(\times$, dotted line) and PAA silicated hematite NPs ( $\square$, solid line). Particle concentration is $0.01 \mathrm{~g} / \mathrm{L}$ and $\mathrm{pH}=7$. The vertical lines show the critical limit for the salt concentration (ccc) above which aggregation occurs.

observed (Figure 5A). These results are in agreement with work on hematite nanoparticles by Baalousha ${ }^{14}$ and Zhang et al. ${ }^{17}$ Independent of the presence of PAA, silicated hematite NPs both with and without PAA show an isoelectric point at $\mathrm{pH}=4$. Therefore, the silica layer increases the charge density at neutral $\mathrm{pH}$, which leads to a higher electrophoretic mobility under these conditions. The significantly enhanced electrostatic repulsion of particles at $\mathrm{pH}>5$ thus results in the formation of stable aqueous suspensions of modified nanoparticles. PAA has a $\mathrm{p} K_{\mathrm{a}}$ of 4 and, therefore, the polyelectrolyte coating does not alter the isoelectric point. However, the polymer does influence the aggregation behavior. When $\mathrm{pH}$ decreases from 7 to 2 , the coated particles aggregate at the isoelectric point as shown by the increase of hydrodynamic radius in Figure 5B. However, while the silica-coated NPs stay aggregated at $\mathrm{pH}$ below the isoelectric point, the hydrodynamic radius of PAA-silica-coated NPs returns to the initial value found for individual nanoparticles. This result is confirmed by four repeated $\mathrm{pH}$ variations from 7 to 2 and 2 to 7 on the same suspension and demonstrates that aggregation is reversible in the presence PAA. It clearly indicates that the presence of an additional PAA layer results in a steric contribution to the interaction potential, which then renders the $\mathrm{pH}$-induced aggregation at the isoelectric point reversible.

At neutral $\mathrm{pH}$, the effect of the addition of salt on the aggregation of coated particles was monitored with DLS. Figure 6 shows the evolution of the hydrodynamic radius with increasing salt concentration for the silicated hematite particles with and without PAA. Without PAA, aggregation starts when the salt concentration reaches about $40 \mathrm{mM}$. In the presence of PAA, three regimes can be distinguished: (A) For $[\mathrm{KCl}]<50 \mathrm{mM}$, the hydrodynamic radius $R_{\mathrm{h}}=77 \pm 7 \mathrm{~nm}$ is constant and the suspension is stable; (B) for $50 \mathrm{mM}<[\mathrm{KCl}]<100 \mathrm{mM}, R_{\mathrm{h}}$ increases gradually to about $100 \mathrm{~nm}$; (C) for $[\mathrm{KCl}]>100 \mathrm{mM}$, the salt now completely screens the electrostatic repulsion, the particles form clusters, and sedimentation sets in. This study clearly shows considerable improvements in the particle stability due to the combined PAA-silica shell. Our innovative surface coating process allows working under conditions which approach the ionic strength and $\mathrm{pH}$ values encountered in biological applications.

While salt-induced aggregation in NP suspensions is generally irreversible, the addition of a PAA layer leads to reversible aggregation for $\mathrm{PAA}-\mathrm{SiO}_{2}$-hematite NPs. This was demonstrated by a series of experiments where aggregation was first induced by the addition of $200 \mathrm{mM}$ salt, aggregation was allowed to proceed during an induction period of $30 \mathrm{~min}$, and then the particles were washed by four cycles of centrifugation and redispersed in pure water. Throughout these different steps, the aggregation state was monitored by DLS. The thus-obtained correlation functions from the suspensions before and after the addition of salt and after washing are shown in Figure $4 \mathrm{~S}$ in the Supporting Information. Initially, a value of $R_{\mathrm{h}}=110 \pm 5 \mathrm{~nm}$ was obtained for the stable dispersion. In the presence of $200 \mathrm{mM}$ of $\mathrm{KCl}$, aggregation sets in and $R_{\mathrm{h}}$ increases and reaches a value of $200 \mathrm{~nm}$ after an induction period of $30 \mathrm{~min}$. After the repeated washing procedure, the DLS measurements on the redispersed samples result in the value $R_{\mathrm{h}}=130 \pm 10 \mathrm{~nm}$, i.e., very close to the initial value obtained for the stable suspension. This clearly indicates that the salt-induced aggregation was fully reversible in the case of the $\mathrm{PAA}-\mathrm{SiO}_{2}$-hematite NPs, and that after washing and redispersion only a small number of clusters remain.

\section{CONCLUSION}

We have reported on a single-step coating method that allows us to prepare anisotropic magnetic nanoparticles with a mixed silicaPAA shell. It is based on an established silica coating procedure, but in the presence of PAA. An extensive characterization of the resulting particles using a combination of TEM, infrared spectroscopy, and SAXS has clearly demonstrated that this synthesis protocol results in the formation of a silica shell with a thickness of about $10 \mathrm{~nm}$ with partially incorporated PAA chains. DLS and electrophoretic light scattering have shown that the coating shifts the isoelectric point away from neutral $\mathrm{pH}$, provides additional electrosteric stabilization, and thus significantly enhances the stability of the dispersion against the addition of salt. The new hybrid coating allows working under $\mathrm{pH}$ and salt conditions that approach those encountered in biological applications. Moreover, in contrast to a silica coating that also enhances the stability of the NPs through a purely electrostatic stabilization mechanism at neutral $\mathrm{pH}$, the additional PAA layer adds an electrosteric stabilization mechanism that renders salt or $\mathrm{pH}$-induced aggregation reversible, thus allowing flocculation and redispersion of the particles in a completely reversible way. It is important to point out that this simple and efficient coating procedure is not limited to the hematite particles used in this study. As the applied synthesis method has been inspired by a well-established general method, ${ }^{34}$ it is thus not limited to bare hematite particles but can be adapted to the entire class of silicacoatable particles. This opens up interesting and promising perspectives to develop enhanced suspension stability for a broad range of colloids through electrosteric shell structures using a single-step synthesis.

\section{ASSOCIATED CONTENT}

S Supporting Information. Additional figures showing nanoparticle characterization (size distribution of hematite nanoparticles; infrared spectra before and after the coating step and after three months of storage), and stability study (DLS correlation functions for coated particles with, without salt and after 
removal of salt).

\section{AUTHOR INFORMATION}

\section{Corresponding Author}

${ }^{*}$ Dr. Hervé Dietsch, Adolphe Merkle Institute and Fribourg Center for Nanomaterials, University of Fribourg, Marly 1, CH-1723 (Switzerland); E-mail herve.dietsch@unifr.ch; Tel + 4126300 9137; Fax + 4126300 9624. Prof. Dr. Peter Schurtenberger, Physical Chemistry, University of Lund, S-22100 Lund, Sweden; E-mail peter.schurtenberger@fkem1.lu.se.

\section{ACKNOWLEDGMENT}

The small-angle X-ray scattering experiments were performed at the cSAXS beamline of the Swiss Light Source at the Paul Scherrer Institute (Villigen, Switzerland), the authors thank in particular A. Menzel for his expert help during the measurements. We also acknowledge financial support for the project provided by the Adolphe Merkle Foundation, COST action D43 and the Swiss State Secretariat for Education and Research, and the Swiss National Science Foundation.

\section{REFERENCES}

(1) Gupta, A. K.; Gupta, M. Biomaterials 2005, 26 (18), 3995-4021.

(2) Ocaña, M.; Morales, M. P.; Serna, C. J. J. Colloid Interface Sci. 1999, $212(2), 317-323$.

(3) Lee, S. H.; Song, Y.; Hoseina, I. D.; Liddell, C. M. J. Mater. Chem. 2009, 19, 350-355.

(4) Ding, T.; Liu, Z. F.; Song, K.; Tung, C. H. Colloids Surf., A 2009, $336(1-3), 29-34$

(5) Hajdu, A.; Tombácz, E.; Illés, E.; Bica, D.; Vékás, L. Prog. Colloid Polym. Sci. 2008, 135, 29-37.

(6) Liu, L.-H.; Dietsch, H.; Schurtenberger, P.; Yan, M. Bioconjugate Chem. 2009, 20 (7), 1349-1355.

(7) Bahadur, D.; Giri, J. Sadhana 2003, 28, 639-656.

(8) Saiyed, Z. M.; Telang, S. D.; Ramchand, C. N. BioMag. Res. Technol. 2003, 1 (1), 2.

(9) Pankhurst, Q. A.; Thanh, N. K. T.; Jones, S. K.; Dobson, J. J. Phys. D: Appl. Phys. 2009, 42 (22), 15.

(10) Shukoor, M. I.; Natalio, F.; Tahir, M. N.; Ksenofontov, V.; Therese, H. A.; Theato, P.; Schroder, H. C.; Muller, W. E. G.; Tremel, W. Chem. Commun. 2007, No. 44, 4677-4679.

(11) Mahmoudi, M.; Simchi, A.; Imani, M. J. Phys. Chem. C 2009, 113 (22), 9573-9580.

(12) Hilger, I.; Hergt, R.; Kaiser, W. A. J. Magn. Magn. Mater. 2005, 293 (1), 314-319.

(13) Reufer, M.; Dietsch, H.; Gasser, U.; Grobety, B.; Hirt, A. M.; Malik, V. K.; Schurtenberger, P. J. Phys.: Condens. Matter 2011, 23 (6), 065102.

(14) Baalousha, M. Sci. Total Environ. 2009, 407, 2093-2101.

(15) Phenrat, T.; Saleh, N.; Sirk, K.; Tilton, R. D.; Lowry, G. V. Environ. Sci. Technol. 2006, 41 (1), 284-290.

(16) Plaza, R. C.; Quirantes, A.; Delgado, A. V. J. Colloid Interface Sci. 2002, 252 (1), 102-108.

(17) Zhang, J.; Huguenard, C.; Scarnecchia, C.; Menghetti, R.; Buffle, J. Colloids Surf., A 1999, 151 (1-2), 49-63.

(18) Tiraferri, A.; Chen, K. L.; Sethi, R.; Elimelech, M. J. Colloid Interface Sci. 2008, 324 (1-2), 71-79.

(19) Zelenev, A.; Matijevic, E. J. Colloid Interface Sci. 2006, 299 (1), 22-27.

(20) Fauconnier, N.; Bée, A.; Roger, J.; Pons, J. N. J. Mol. Liq. 1999, $83,233-242$
(21) Lee, J.; Isobe, T.; Senna, M. J. Colloid Interface Sci. 1996, 177 (2), 490-494.

(22) Nath, S.; Kaittanis, C.; Ramachandran, V.; Dalal, N. S.; Perez, J. M. Chem. Mater. 2009, 21 (8), 1761-1767.

(23) Zhang, T.; Ge, J.; Hu, Y.; Yin, Y. Nano Lett. 2007, 7 (10), 3203-3207.

(24) Caruso, F.; Spasova, M.; Susha, A.; Giersig, M.; Caruso, R. A. Chem. Mater. 2000, 13 (1), 109-116.

(25) Mohanty, P. S.; Dietsch, H.; Rubatat, L.; Stradner, A.; Matsumoto, K.; Matsuoka, H.; Schurtenberger, P. Langmuir 2009, 25 (4), 1940-1948.

(26) Haq, I. u.; Matijevic, E. J. Colloid Interface Sci. 1997, 192 (1), $104-113$

(27) Ohmori, M.; Matijevic, E. J. Colloid Interface Sci. 1992, 150 (2), 594-598.

(28) Wenru, Z.; Hangrong, C.; Yongsheng, L.; Liang, L.; Meidong, L.; Jianlin, S. Adv. Funct. Mater. 2008, 18 (18), 2780-2788.

(29) Wang, S. F.; Cao, H. M.; Gu, F.; Li, C. Z.; Huang, G. J. J. Alloys Compd. 2008, 457 (1-2), 560-564.

(30) Zou, H.; Wu, S.; Shen, J. Chem. Rev. 2008, 108 (9), 3893-3957.

(31) Tsang, S. C.; Yu, C. H.; Gao, X.; Tam, K. J. Phys. Chem. B 2006, 110 (34), 16914-16922.

(32) Butterworth, M. D.; Bell, S. A.; Armes, S. P.; Simpson, A. W. J. Colloid Interface Sci. 1996, 183 (1), 91-99.

(33) Dagallier, C.; Dietsch, H.; Schurtenberger, P.; Scheffold, F. Soft Matter 2010, 6, 2174-2177.

(34) Graf, C.; Vossen, D. L. J.; Imhof, A.; van Blaaderen, A. Langmuir 2003, 19 (17), 6693-6700.

(35) Sacanna, S.; Rossi, L.; Kuipers, B. W. M.; Philipse, A. P. Langmuir 2006, 22 (4), 1822-1827.

(36) Wang, Y.; Angelatos, A. S.; Dunstan, D. E.; Caruso, F. Macromolecules 2007, 40 (21), 7594-7600.

(37) Sacanna, S.; Rossi, L.; Wouterse, A.; Philipse, A. P. J. Phys.: Condens. Matter 2007, 19 (37), 16.

(38) Reufer, M.; Dietsch, H.; Gasser, U.; Hirt, A.; Menzel, A.; Schurtenberger, P. J. Phys. Chem. B 2010, 114 (12), 4763-4769. 